\title{
Estratégia para o tratamento cirúrgico das anomalias da conexão ventrículo-arterial com comunicação interventricular
}

\author{
José CALIANI*, Yves LECOMPTE*
}

RBCCV 44205-87

CALIANI, J. \& LECOMPTE, Y. - Estratégia para o tratamento cirúrgico das anomalias da conexão ventrículo-arterial com comunicação interventricular. Rev. Bras. Cir. Cardiovasc., 4(2): 159-162, 1989.

RESUMO: Designamos anomalias da conexão ventrículo-arterial toda a conexão que difere daquela de um coraçāo normal. Dentro desta abordagem, não utilizamos os termos ventrículo direito ou esquerdo com dupla via de saída, Taussig-Bing e transposição das grandes artérias, quando estes estão associados a uma comunicação interventricular. Nestas anomalias, o objetivo da correçâo é o de conectar o ventrículo esquerdo com a aorta e o ventrículo direito com a artéria pulmonar. A estratégia que escolhemos é baseada na hipótese de que a correção mais simples é aquela que não exige a utilização de um tubo protético, a transferência de coronárias, ou a septação complexa da cavidade ventricular. Baseados na experiência de 162 correçōes para as anomalias da conexão ventrículo-arterial, em uma série de 197 pacientes, utilizamos três tipos de correçăo anatômica: a correção intraventricular (tunelização ventrículo esquerdo-aorta) em 35 pacientes, o REV (tunelização ventrículo esquerdo-aorta associada a translocaçāo do tronco pulmonar sobre o ventrículo direito) em 78 pacientes, e a operação de Jatene associada ao fechamento da comunicação interventricular em 49 pacientes. O tipo de correção ideal é a correçăo intraventricular, na qual a simples tunelizaçăo ventrículo esquerdo-aorta estabelece uma conexão ventrículo-arterial normal. Quando a correção intraventricular năo é possível, nós indicamos o REV em presença de estenose pulmonar e a operaçăo de Jatene na ausência desta. A questão principal é saber quando uma correção intraventricular é realizável. A realização desta é função da distância entre a valva tricúspide e a valva pulmonar. Se esta distância é suficientemente grande (igual ou superior ao diâmetro do orifício aórtico), o túnel intraventricular é realizável; se năo, outra modalidade de correçăo é indicada. Nossa experiência atual sugere que a exploraçăo pré-operatória das distâncias entre a valva tricúside e as válvulas semilunares é um critério essencial para a escolha da correçāo apropriada para as anomalias da conexão ventrículo-arterial associadas a uma comunicação interventricular. Esta estratégia não se opōe às outras classificaçōes usuais, baseadas na posição das grandes artérias, ou na situação da comunicação interventricular, e ela nos fornece informaçōes precisas quanto à possibilidade de realizar uma correção intraventricular.

DESCRITORES: conexão ventrículo-arterial, cirurgia.

\section{INTRODUÇÃO}

Há quase dois séculos, os coraçōes com conexōes ventrículo-arteriais anormais, em particular o ventrículo direito com dupla via de saida (DVS), eram reconhecidos como formas de cardiopatias congênitas ${ }^{1}$. No que diz respeito às anomalias da conexão ventrículo-arterial (VA) com comunicação interventricular (CIV), inúmeras classificaçōes anatômicas foram propostas, mas estas permanecem insatisfatórias. Por exemplo, a definição de ventriculo direito com DVS é matéria de controvérsia e as diferentes modalidades de correção cirúrgica propostas

Trabalho realizado no Hópital Laënec e Centre Medico-Chirurgicale, Porte de Choisy. Paris, França.

Apresentado ao 16: Congresso Nacional de Cirurgia Cardiaca. Sảo Paulo, 7 e 8 de abril, 1989.

- Do Hópital Laënec e Centre Medico-Chirurgicale, Porte de Choisy.

Endereço para separatas: J. Caliani. Centre Medico-Chirurgicale de la Porte de Choisy. 6, Place de Port au Prince 75013 Paris, France. 
CALIANI, J. \& LECOMPETE, Y. - Estratégia para o tratamento cirúrgico das anomaliał da conexão ventrículo-arterial com comunicação interventricular. Rev. Bras. Cir. Cardiovasc., 4(2): 159-162, 1989.

para esta lesăo podem, também, ser utilizadas para outras cardiopatias ${ }^{10}$. Por estas razōes, preferimos incluir, em nossa estratégia cirúrgica, todas as anomalias da conexão VA associadas a uma CIV e com conexão atrioventricular normal. O objetivo deste trabalho é definir os critérios anatômicos que seriam úteis para a escolha do melhor tipo de correção para as anomalias da conexão VA associadas a uma CIV, com ou sem estenose da via pulmonar.

\section{DEFINIÇŌES E CRITÉRIOS PARA A CONDUTA CIRÚRGICA}

Designamos anomalias da conexão VA toda a conexão que difere daquela de um coração normal. Nesta definição estão incluídas as anomalias usualmente denominadas ventrículo direito ou esquerdo com DVS, Taussig-Bing e transposiçāo das grandes artérias associada a uma CIV.

Atualmente, utilizamos três tipos de correçăo anatômica para o tratamento das anomalias da conexāo VA:

1) Correção intraventricular (RIV) - Nesta modalidade, incluem-se todos os tipos de correção que estabeleçam uma conexão VA normal com a simples tunelização ventrículo esquerdo-aorta. Na maioria dos casos, esta correção deve ser complementada por uma ressecção septal (septo conal e/ou parte do septo que está localizado anteriormente à CIV). O fechamento de uma CIV representa o tipo mais simples de correção intraventricular, sendo que as técnicas descritas por KIRKLIN et alii $^{4}$ para a correção do clássico ventrículo direito com DVS, e por KAWASHIMA et alii ${ }^{3}$ para o tratamento do Taussig-Bing são tipos mais complexos de correçōes intraventriculares. Nós năo temos experiência com as técnicas descritas por PATRICK \& $\mathrm{McGOON}^{8}$ e, mais recentemente, por PACÍFICO et aliii ${ }^{7}$. Estas últimas não estã incluídas em nossa definição de correção intraventricular.

2) REV Reparation à l'étage ventriculaire - Originalmente descrita por LECOMPTE et alii ${ }^{5}$, esta é, por definição, a associação da correção interventricular com a translocaçăo do tronco pulmonar sobre o ventrículo direito.

3) Operação de JATENE ${ }^{2}-O$ princípio desta técnica é o de conectar o ventrículo esquerdo com o orifício pulmonar e realizar, ao nível arterial, uma troca das grandes artérias com a transferência dos óstios coronarianos do seio de Valsalva aórtica para o tronco pulmonar. Em nossa série, realizamos a manobra de LECOMPTE $^{6} \mathrm{em}$ todos os casos

A estratégia cirúrgica está contida em duas questōes fundamentais:

1) Quais são as condiçōes necessárias para que uma correção anatômica possa ser realizada?
2) Se estas condiçōes preliminares forem satisfeitas, qual dos três tipos de correção anatómica deve ser escoIhido?

Respondendo a estas questōes, elaboramos uma estratégia cirúrgica, recentemente descrita ${ }^{9}$ e cujos princípios serăo apresentados abaixo.

a) Condições necessárias para uma correçăo anatômica - Ambos os ventrículos devem ser normais em tamanho e funçăo, e a pressāo do ventrículo esquerdo deve ser sistêmica. Na avaliaçăo pré-operatória, é difícil medir as dimensões das cavidades ventriculares e acreditamos que a medida do diâmetro da valva atrioventricular seja um critério confiável. Assim sendo, uma valva atrioventricular hipoplásica (dois pontos abaixo do diâmetro teórico) é uma contra-indicação à correção anatômica. Outra condição importante é a exclusão de comunicaçōes interventriculares múltiplas. Finalmente, a idade, o estado nutricional e a funçāo respiratória devem ser considerados antes da conduta cirúrgica.

b) Escolha do tipo de correção - Por razōes empíricas, preferimos a correção intraventricular, e a realização desta é funçăo das distâncias entre a valva tricúspide e as valvas semilunares. Estas distâncias săo medidas em ecografia bidimensional e graduadas de 0 a 3 . Cada caso de anomalia da conexão AV pode ser representado por dois números: o primeiro representa a mínima distância entre a valva tricúspide e a valva aórtica, o segundo a distância entre a valva tricúspide e a valva pulmonar. Quando a distância entre a valva tricúspide e pulmonar é suficientemente grande (igual a 3 , ou igual ou superior ao diâmetro do orifício aórtico), a correçăo intraventricular é realizável com ou sem ressecçăo septal e/ou alargamento da via pulmonar. Nos outros casos, realizamos o REV em presença de estenose pulmonar e a operação de Jatene, na ausência desta. Nesta última categoria de pacientes, a escolha cirúrgica é feita baseada em consideraçōes fisiológicas: o REV, ainda que anatomicamente realizável, deve ser evitado pelo alto risco de regurgitação pulmonar e insuficiência ventricular direita.

\section{CASUÍSTICA E MÉTODOS}

De janeiro de 1979 a novembro de 1988,197 pacientes foram submetidos a 327 operaçōes para as anomalias da conexão VA com CIV. Do total das operaçōes 167 foram correçōes anatómicas e as demais correspondem a intervençōes paliativas (Gráfico 1). A avaliaçăo pré-operatória e as indicaçōes cirúrgicas foram baseadas nos critérios ecográficos acima descritos.

A idade variou do período neonatal até 12 anos, refletindo a ausência de homogeneidade deste grupo (Gráfico 2). Algumas anomalias associadas foram encontradas: nove coarctaçōes ou interrupçōes do arco aórtico, 12 CIVs múltiplas e nove hipoplasias das valvas atrioventriculares. 

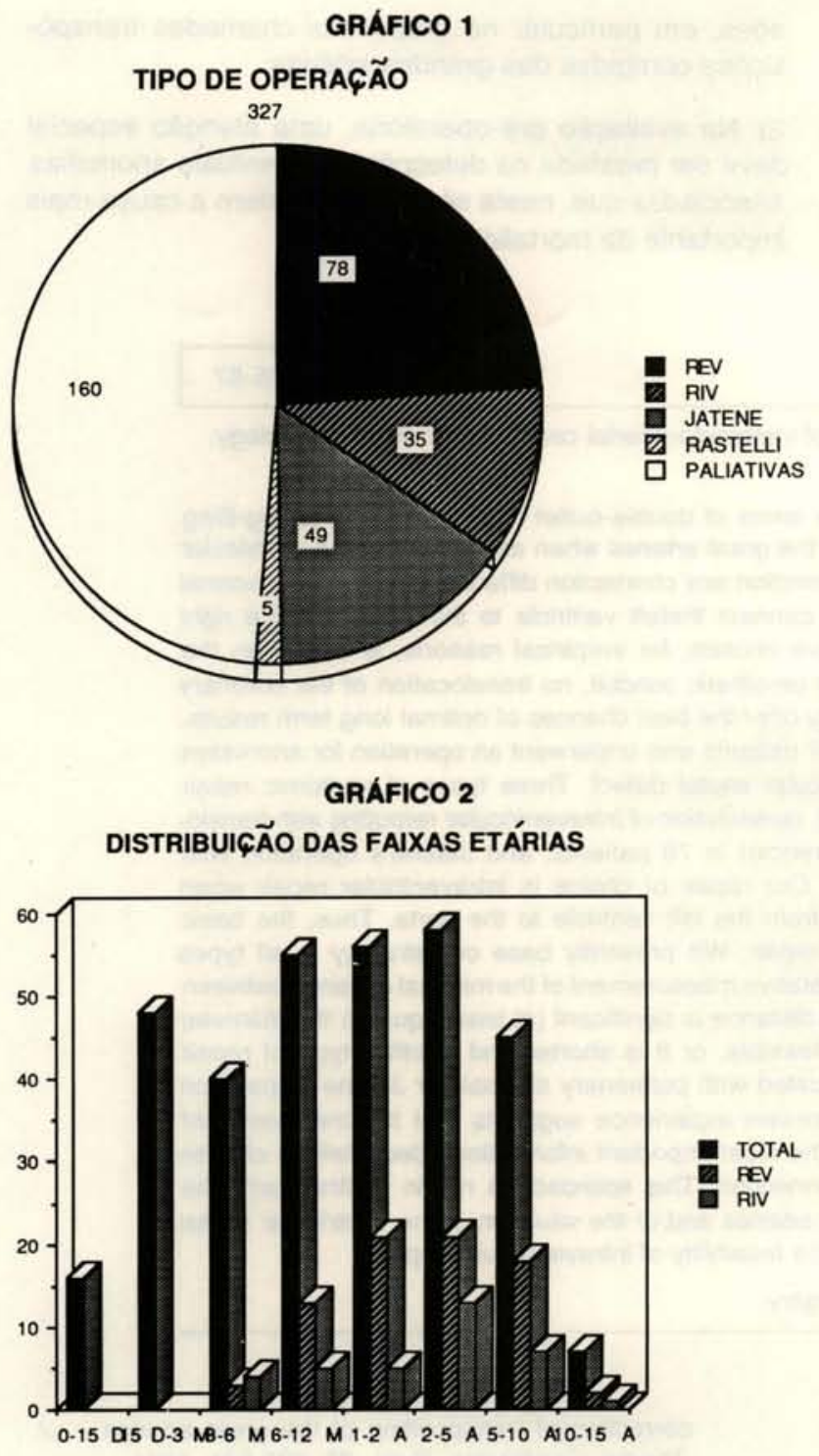

\section{RESULTADOS}

A correção anatômica foi contra-indicada em 30 pacientes. Dentre estes, 15 apresentavam ao menos uma contra-indicação absoluta; nos demais, a idade e a presença de anomalias associadas nos conduziram a praticar operações paliativas.

Em cinco das 167 correçōes anatômicas foi realizada a operaçāo de Rastelli, que, segundo nossa definição, não está de acordo com o termo "correção anatômica", porque esta requer a utilização de um conduto protético. Dos cinco pacientes, três foram submetidos a operaçōes prévias em outros centros e a presença de aderências mediastinais nos impossibilitou a mobilização do tronco pulmonar. Em outro caso, um conduto, valvado foi indicado na presença de uma resistência pulmonar elevada e, no último paciente, uma ventriculotomia ampla nos abrigou a utilizar um conduto protético a fim de proteger uma das artérias coronárias. Com excessão destes cinco pacientes, todos os demais se beneficiaram de um dos três tipos de correção anatômica descritos na primeira parte deste trabalho.

A mortalidade global foi de $11,3 \%$ (37 pacientes para 327 operaçōes), sendo que 15 óbitos ocorreram nos pacientes submetidos à operação de Jatene, 13 na série do REV, três na série da correção intraventricular e os seis demais no grupo das paliativas (Gráfico 3 ). Dentre as causas de óbito, constatamos um número significativo de anomalias associadas, que foram subestimadas nas exploraçōes pré-operatórias, sendo as mais freqüentes a hipoplasia de um dos ventrículos (cinco pacientes) e as CIVs múltiplas (cinco pacientes). Onze óbitos não estão relacionados com a estratégia cirúrgica: erros técnicos (cinco pacientes), mediastinite (três pacientes), hemoptise massiva (um paciente), hemorragia intra-operatória (um paciente) e hemorragia cerebral (um paciente). Um dos pacientes faleceu de insuficiência ventricular direita aguda, após a realização de manobra de Lecompte; isto deveu-se ao fato de que este não apresentava estenose da via pulmonar e a regurgitação causada pela manobra acabou sendo mal tolerada pelo ventrículo direito. Dois óbitos ocorreram tardiamente, o primeiro de uma arritmia ventricular um ano após a operação; o segundo evoluiu com insuficiência cardiaca global; em seguida, foi transplantado e faleceu de rejeição, na unidade de terapia intensiva. Em 12 pacientes, não foi possível identificar, com precisão, a causa do óbito. Em um dos pacientes, a extratégia foi, provavelmente, inadequada. Este apresentava uma anomalia da conexão VA do tipo 3-3 (em outra terminologia, Taussig-Bing) e se beneficiou de uma correção intraventicular. Uma semana após tal evento, veio a falecer de uma rotura septal, sendo que a tunelização ventrículo esquerdo-aorta estava anatomicamente intacta. A causa desta rotura foi, possivelmente, devida à ressecção septal, que, neste caso, foi bastante ampla. Retrospectivamente, acreditamos que, para este paciente, teria sido melhor realizar a operação de Jatene.

\section{GRÁFICO 3}

MORTALIDADE OPERATÓRIA

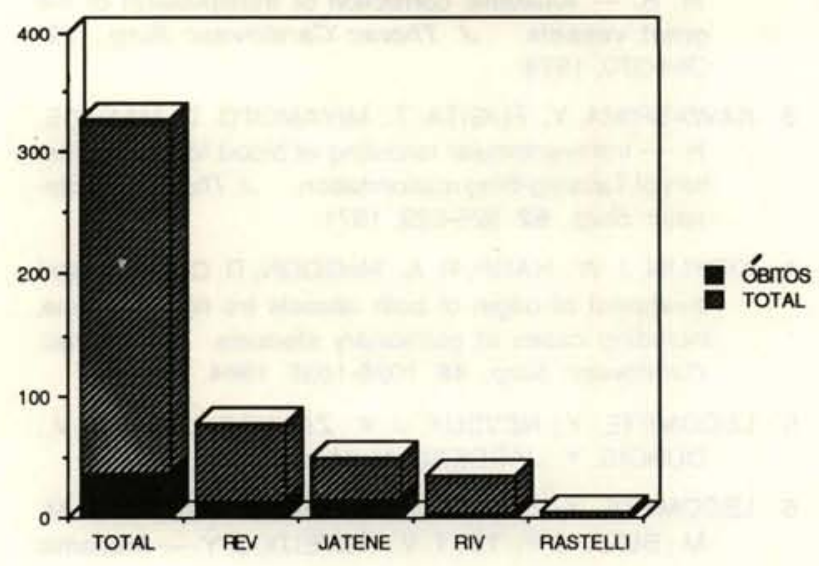


CALIANI, J. \& LECOMPETE, Y. - Estratégia para o tratamento cirúrgico das anomalias da conexão ventrículo-arterial com comunicação interventricular. Rev. Bras. Cir. Cardiovasc., 4(2): 159-162, 1989.

\section{CONCLUSĀO}

Nossa experiência atual fornece duas conclusões:

1) A estratégia escolhida para o tratamento cirúrgico das anomalias da conexão VA é adequada e útil, na maioria dos casos; entretanto, existem algumas exces- sões, em particular no grupo das chamadas transposiçōes corrigidas das grandes artérias.

2) $\mathrm{Na}$ avaliação pré-operatória, uma atenção especial deve ser prestada na deteç̧ăo de eventuais anomalias associadas que, nesta série, representam a causa mais importante da mortalidade hospitalar.

RBCCV 44205-87

CALIANI, J. \& LECOMPTE, Y. - Repair of anomalies of ventriculo-arterial connections: surgical strategy. Rev. Bras. Cir. Cardiovasc., 4(2): 159-162, 1989.

ABSTRACT: In our practice, we no longer use the terms of double-outlet right ventricle, Taussig-Bing anomaly, double outlet left ventricle, or transposition of the great arteries when associated with a ventricular septal defect. We call anomalous ventriculo-arterial connection any connection different from that of a normal heart. In these anomalies the aim of the repair is to connect theleft ventricle to the aorta and the right ventricle to the pulmonary trunk. The strategy we have chosen, for empirical reasons, is based on the hypothesis that the simplest methods which require no prosthetic conduit, no translocation of the coronary arteries and no complex partition of the ventricular cavity offer the best chances of optimal long term results. The feasibility of anatomic repair was investigated in 197 patients who underwent an operation for anomalies of ventriculo-arterial connection associated with ventricular septal defect. Three types of anatomic repair were used: intraventricular rerouting (35 patients), R.E.V. (association of intraventricular rerouting with translocation of the pulmonary arterial trunk on the right ventricle) in 78 patients, and Jatene's operation with closure of the ventricular septal defect (49 patients). Our repair of choice is intraventriclar repair when it is possible to construct a straight and direct tunnel from the left ventricle to the aorta. Thus, the basic question is the feasibility of a simple intraventricular repair. We presently base our strategy in all types of anomalous ventriculo-arterial connection on the preoperative measurement of the minimal distance between the tricuspid valve and the pulmonary valve. Either this distance is significant (at least equal to the diameter of the aortic orifice) and the intraventricular repair is feasible, or it is shorter and another type of repair is indicated. We then perform a R.E.V. in cases associated with pulmonary stenosis or Jatene's operation in cases with increased pulmonary blood flow. Our present experience suggests that the measurement of intervalvar distances is, from a surgical standpoint, the most important information adequately to choose the of repair for the anomalies of ventriculoarterial connection. This approach is not in contrast with the usual classifications based on the position of the great arteries and/or the situation of the ventricular septal defects. It gives information which is directly related to the feasibility of intraventricular repair.

DESCRIPTORS: ventriculo-arterial connection, surgery.

\section{REFERÊNCIAS BIBLIOGRÁFICAS}

1 ABERNETHY, J. - Surgical and physiological essays. London, James, Evans, Publishers, 1973.

2 JATENE, A. D.; FONTES, V. F.; PAULISTA, P. P.; SOUZA, L. C. B.; NEGER, F.; GALANTIER, M.; SOUZA, J. E. M. R. - Anatomic correction of transposition of the great vessels. J. Thorac Cardiovasc Surg., 72: 364-370, 1976

3 KAWASHIMA, Y.; FUGITA, T.; MIYAMOTO, T.; MANABE, H. - Intraventricular rerouting of blood for the correction of Taussig-Bing malformation. J. Thorac. Cardiovasc. Surg., 62: 825-829, 1971.

4 KIRKLIN, J. W.; HARP, R. A.; McGOON, D. C. - Surgical treatment of origin of both vessels fro right ventricle, including cases of pulmonary stenosis. J. Thorac. Cardiovasc. Surg., 48: 1026-1036, 1964.

5 LECOMPTE, Y.; NEVEUX, J. Y.; ZANNINI, L.; TU, T. V.; DUBOIS, Y.; JARREAU, M. M.

6 LECOMPTE, Y; ZANNINI, L.; HAZAN, E.; JARREAU, M. M.; BENX, J. P.; TU, T. V.; NEVEUX, J. Y. - Anatomic correction of transposition of the great arteries. $J$ Thorac. Cardiovasc. Surg., 82: 629-631, 1981.

7 PACÍFICO, A. D.; KIRKLIN, J. W.; COLVIN, E V.; BARGERON, L. M. - Intraventricular tunnel repair for TaussigBing heart and related cardiac anomalies. Circulation, 74:153-160, 1986.

8 PATRICK, D. L. \& McGOON, D. C. - An operation for double-outlet right ventricle with transposition of the great arteries. J. Cardiovasc, Surg., 9: 537-542, 1968.

9 SAKATA, R.; LECOMPTE, Y.; BATISSE, A.; BORROMÉE, L.; DURANDY, Y. - Anatomic repair of anomalies of ventriculo-arterial connection associated with ventricular septal defect: I. Criteria of surgical decision. J. Thorac. Cardiovasc. Surg., 95: 90-95, 1988.

10 WILCOX, B. R.; HO, S. Y.; MACARTNEY, F. J.; BACKER, A. E.; GERLIS, L. M.; ANDERSON, R. H. - Surgical anatomy of double-outlet right ventricle with situs solitus and atrioventricular concordance. J. Thorac. Cardiovasc. Surg., 82: 405-417, 1981. 